\title{
CFD investigations of the effect of rotating wheels, ride height and wheelhouse geometry on the drag coefficient of electric vehicle
}

\author{
A. Alekseev ${ }^{1}$, A. Maksimov, A. Tarasov \\ Peter the Great St. Petersburg Polytechnic University \\ Russia
}

Received: June 17, 2020. Revised: July 20, 2020. Accepted: July 21, 2020. Published: July 27, 2020.

\begin{abstract}
The development of electric vehicles demands minimizing aerodynamic drag in order to provide maximum range. The wheels contribute significantly to overall drag coefficient value because of flow separation from rims and wheel arches. In this paper various design parameters are investigated and their influence on vehicle drag coefficient is presented. The investigation has been done with the help of computational fluid dynamics (CFD) tools and with implementation of full vehicle setup with rotating wheels. The obtained results demonstrate changes in drag coefficient with respect to the change of design parameters.
\end{abstract}

Keywords-Drag coefficient, electric vehicle, rotating wheels, vehicle aerodynamics.

\section{INTRODUCTION}

$\mathrm{I}^{\mathrm{r}}$ $\mathrm{n}$ the light of preventing further increase of global warming and sustainable environment trends in the society the international and country authorities are introducing measures to decrease $\mathrm{CO}_{2}$ emissions. These measures push the auto makers to develop and bring to the market new and efficient electric vehicles, as conventional cars driven by internal combustion engines account for approximately $15 \%$ of carbon dioxide emissions in Europe [1]. One of the main challenges in designing an electric vehicle is providing sufficient range for a given battery installation, as there are many other energy consumers onboard the vehicle, such as air conditioning system, external and internal lightning, active and passive safety systems and others. As the overall energy source of the battery is limited, optimization of every car component is of crucial importance. That is why the aerodynamics studies of electric vehicles are becoming important again. Due to the

${ }^{1}$ Corresponding author: alekseev.a@compmechlab.ru

The work was performed with financial support of the Ministry of Science and Higher Education of the Russian Federation as part of the implementation of the Federal Target Program "Research and development in priority areas of the scientific and technological complex of Russia for 2014-2020". Unique identifier of the agreement RFMEFI57818X0269. battery installation normally at the bottom of the vehicle, the underflow becomes almost flat comparing to combustionengine cars, which has positive effect on overall vehicle aerodynamics. At the same time, the rotating wheels and wheel arches significantly contribute to the drag of a vehicle, accounting for up to $25 \%$ of overall drag coefficient value as has been demonstrated by various researchers [2]-[5]. Design of rims and wheel arches determines the intensity of vortex shedding and separation zones and participates in the creation of wake structure. Some previously done researches demonstrate the importance of wheel design configurations and their influence on aerodynamic performance [6]-[7].

The study of car's aerodynamics at early design stages is normally done with the help of CFD, though the design of wheel rims and arches are defined at later phases. Nevertheless CFD tools can help designers to choose between numerous pre-calculated design solutions when the time comes for decision making. This project is a part of electric vehicle development, in which preliminary parametric studies have been done on vehicle aerodynamic drag with respect to the change of rim design, ride height, wheel arch height and various wheel rotation speeds.

\section{Methodology}

The computational domain, which is depicted in Figure 1, is large enough to prevent influence of borders on the resulting solution. It is a rectangular box, which is $34 \mathrm{~m} \mathrm{long,} 14 \mathrm{~m}$ high and $12.5 \mathrm{~m}$ wide. The vehicle is positioned so that there is distance of two car lengths in front of the vehicle. The distance behind the vehicle is taken as eight car lengths for the purpose of proper wake resolution and avoidance of air inflow at outlet boundary. The vehicle cross-sectional area is $2.195 \mathrm{~m}^{2}$, which makes the blockage factor of $1.25 \%$. The computer aided design (CAD) model is cleaned and made watertight, the surface of the vehicle is prepared and optimized for meshing. The surface resolution is done with unstructured tetrahedral mesh of varying size from $5 \mathrm{~mm}$ in the regions of close proximities or high surface curvature to $20 \mathrm{~mm}$ on flat surfaces. Preliminary studies on hexa-core mesh change the 
results significantly. For the purpose of boundary layer resolution the prism mesh layers are built on the vehicle and wheel surfaces with the first layer thickness of $0.02 \mathrm{~mm}$ with the aim of obtaining $\mathrm{y}+$ close to unity. The contact patch between the wheel and the road is modified, so that the layers could be built without excessive squeeze or necessity for collapsing them. The total number of prism layers is 18 with the growth factor of 1.5 resulting in smooth transition from surface to volume mesh, providing last layer aspect ratio of 0.9 . In the vicinity of the car surface and in the expected regions of wake development a few mesh refinement options are introduced, limiting the maximum cell size to $20 \mathrm{~mm}$ just around the car and between $40 \mathrm{~mm}$ and $100 \mathrm{~mm}$ in the wake region. This configuration results in the total cell count of approximately $25 \mathrm{mln}$ cells with minor differences depending on the rim and arch design cases.

The solution is obtained with ANSYS CFX solver. The flow is assumed to be incompressible and isothermal with the physical properties of air at 25 degrees Celsius. The nature of the flow is turbulent and thus solved within Reynolds-averaged Navier-Stokes (RANS) assumption with Shear Stress Transport (SST) being set as turbulence model. The advection term discretization is High Resolution scheme of second order accuracy. For CFX False-Transient Algorithm the fluid timescale is chosen as automatically based on the inlet velocity value with the specified length scale of car length. The root mean square (RMS) residual target is $10^{-4}$ and in all cases the solution run is set for 1500 iterations to reach the desired residuals value and to stabilize the force on the vehicle surface over the last few hundred iterations.

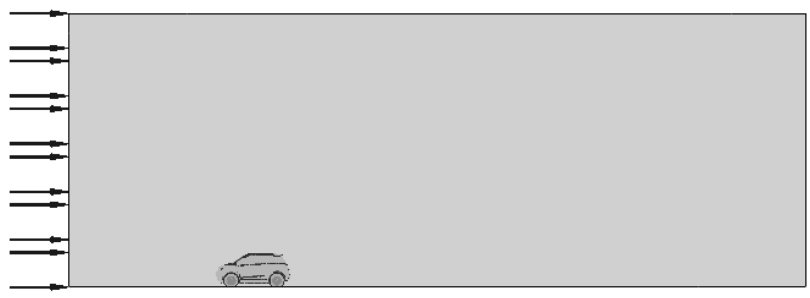

Fig. 1. Simulation domain.

The inlet is a uniform velocity profile normal to the boundary with the velocity value corresponding to the speed of the car in the range between 60 and $120 \mathrm{kph}$ and inlet turbulence intensity of $5 \%$. The outlet is prescribed with average static pressure of $0 \mathrm{~Pa}$ and pressure profile blend of $5 \%$. The moving ground is modeled via assigning wall velocity equivalent to the car speed. Side and top walls of virtual aerodynamic wind tunnel are set to symmetry boundary conditions.

The wheel rotation modeling is implemented with Multiple Reference Frame approach in the zones around wheels with Frozen Rotor interfaces between wheel and external domains [8]. The MRFg approach proposed in [9]-[10] was not considered for the project, as the tires configurations are slick and previous studies of [11] have demonstrated the accuracy of drag prediction with this method in the range of up to one hundred drag counts, which is not necessary for the purpose of the electric vehicle aerodynamics performance at early design stages. The implementation of Sliding Mesh for wheel rotation [12] was not applied because of the presence of the contact patch between tire and road.

\section{SimULATION CASES}

The vehicle in this CFD study is a small two-door car sharing vehicle. An isometric view of the car is shown in Figure 2. The vehicle is modeled without rear view mirrors as their CAD design was not available at early design stage of this electric car. In all cases the simulations are performed for three velocities of 60,90 and $120 \mathrm{kph}$ with corresponding wheel angular rotation speed.

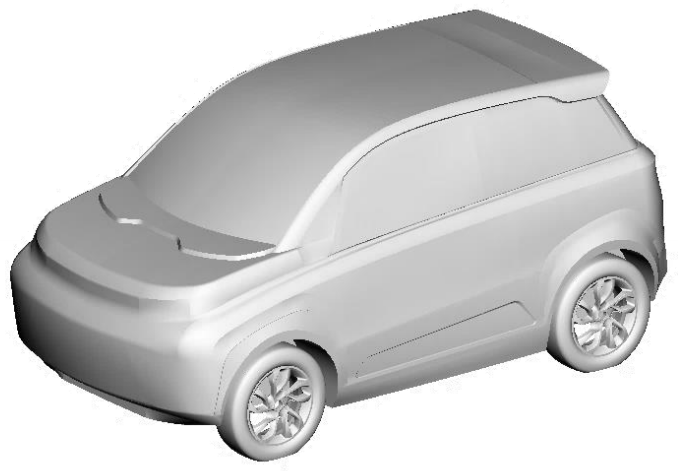

Fig. 2. Design of electric vehicle

Three rim designs are considered: one closed flat rim design (wheel0) and two open rim designs (wheel1 and wheel2). In all cases the tire details like rain and side grooves are not simulated and simulations are implemented on slick tire. The geometries of wheels are presented in Figure 3.
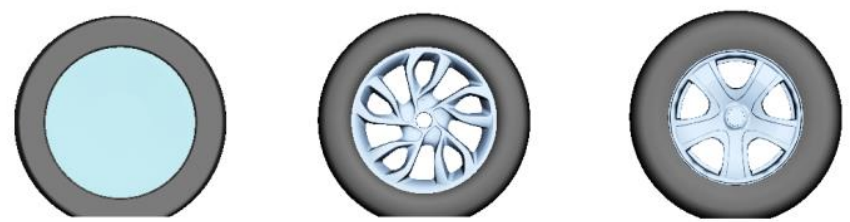

Fig. 3. Rim designs for CFD simulations

Considering $H$ as initial ground clearance, the parametric study of influence of ride height on drag coefficient is implemented in the range between $H$ and $1.5 H$ with the increment of $10 \%$ for each simulation. The two representative ride heights are depicted in Figure 4. 


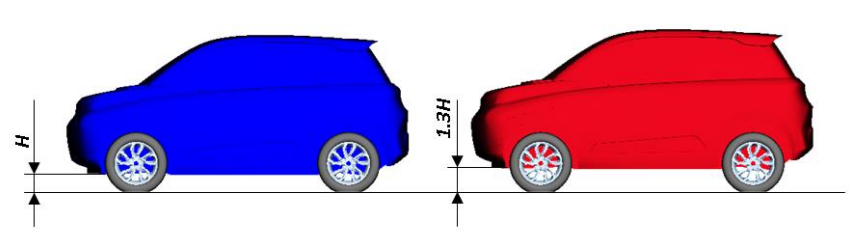

Fig. 4. Ride height change

The morphing of the height of the wheel arches is implemented for four values, starting from base wheel arch height $h$ and reducing down to $0.6 l$ with increment of $10 \%$ each time. It should be noted that the case of $0.6 l$ is implemented on front wheel arches only because of geometrical constraints and necessity to resolve the space between tire surface and wheel arch with volume mesh. The illustration of what is being considered as $h$ is clarified in Figure 5.

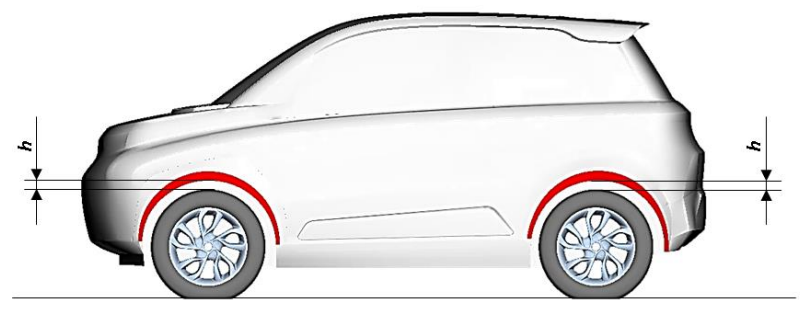

Fig. 5. Wheel arch morphing

\section{RESUlTS AND Discussion}

In this section the results are presented with respect to the influence of corresponding design parameters. The drag coefficient values for various rim designs are shifted by some constant value. The real drag coefficients are not provided but the trends are discussed. This information can be used further by designers when considering wheel rotation effects. When the influence of ride height and wheel arch morphing is investigated, the drag coefficient difference between initial value and value at that design point is specified.

\subsection{Influence of rim design}

Figure 6 summarizes the results obtained from simulations, where wh0, wh1 and wh2 stand for rim design of wheel0, wheell and wheel 2 correspondingly with specified velocity in $\mathrm{kph}$ after underscore. As it can be seen, the case of flat rim design provided minimum drag coefficient value compared to both open rim designs. This can be explained by intensive separation and vortex shedding from rims when open deigns are utilized. The difference is of order of 300 drag counts, which is significant value when making design decisions. Another trend that can be seen is that with increasing velocity and corresponding wheel rotational speed the drag coefficient tend to decrease, though for the case of wheel 2 this trend is less obvious. The non-uniform distribution in trends values can also be attributed to the inaccurate representation of drag coefficient because of averaging over the last few hundred iterations. In order to keep the simulation results consistent and for ease of comparison the drag value convergence histories is not presented in the results.

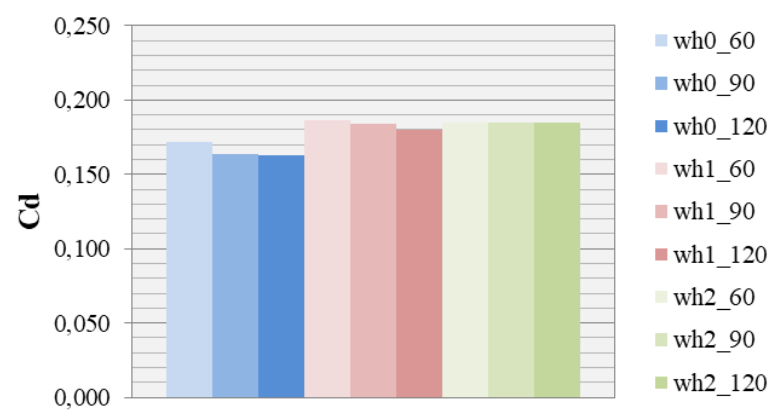

Fig. 6. Influence of rim design on drag

\subsection{Influence of ground clearance}

Figure 7 presents the simulation results obtained from changing the ground clearance. In case of flat rim design there is clear trend of increasing drag coefficient with increasing ride height. This is applicable for each calculated speed of vehicle and wheel rotation. Although the modification of ground clearance of $40 \%$ and $50 \%$ are less likely during the design development, the designer can take into consideration the possible effects on aerodynamic drag in the range of ground clearance increase between $0 \%$ and 30\%. In these cases the increase may take up to 150 drag counts. In case of open rim designs clear drag increasing trend is also applicable for wheel1 design, whereas for wheel2 the trend is less obvious. The increase of drag with higher ground clearance is attributed to the increase of height of the separation zone behind the vehicle, expanding the region of negative pressure. These finding are in correlation with results of ride height influence of [13], in which the drag difference was up to 120 drag counts for even smaller ground clearance elevations.

\subsection{Influence of arch morphing}

Figure 8 reports the simulation results obtained from changing the wheel arch height. In case of flat rim design there is clear trend of drag reduction with corresponding wheel arch height reduction for speeds of $90 \mathrm{kph}$ and $120 \mathrm{kph}$. For the speed of $60 \mathrm{kph}$ drag coefficient also decreased by some constant value compared to non-morphed arch, but reduction trend is not present. Simulations with Wheell design did not provide any clear trends and drug coefficient could increase or decrease between 30 and 50 drag counts. In case of wheel 2 design drag coefficient value decreased in all cases by the value of between 20 and 80 drag counts, though clear trends of decreasing drug with decreasing wheel arch height did not appear. 


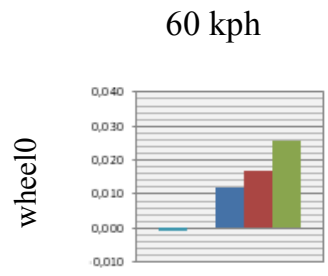

$90 \mathrm{kph}$
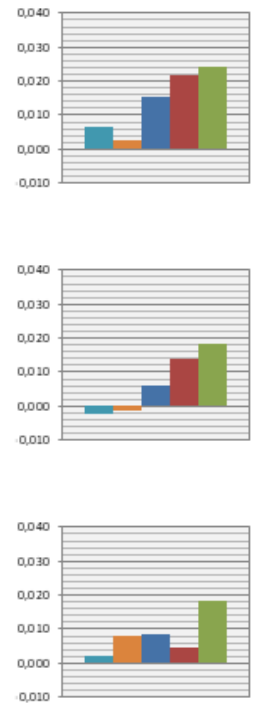

$120 \mathrm{kph}$

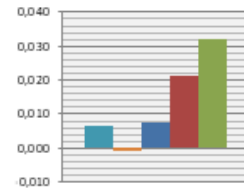

$=\mathrm{GC} 10 \%$

- GC $20 \%$

- GC $30 \%$

= $\mathrm{GC} 40 \%$

- GC $50 \%$

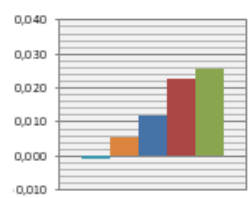

= $\mathrm{GC} 10 \%$

- GC $20 \%$

- GC $30 \%$

a $\mathrm{GC} 40 \%$

GC $50 \%$

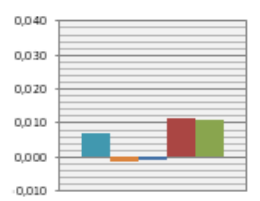

I GC $10 \%$

- GC $20 \%$

- GC $30 \%$

- $\mathrm{GC} 40 \%$

GC 50\%

Fig. 7. Influence of ride height on drag coefficient (compared to $0 \%$ ground clearance)
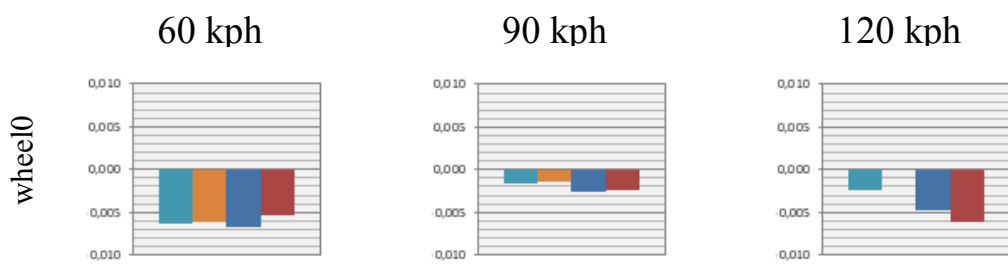

arch $10 \%$

arch $20 \%$

Arch $30 \%$

- Arch $40 \%$
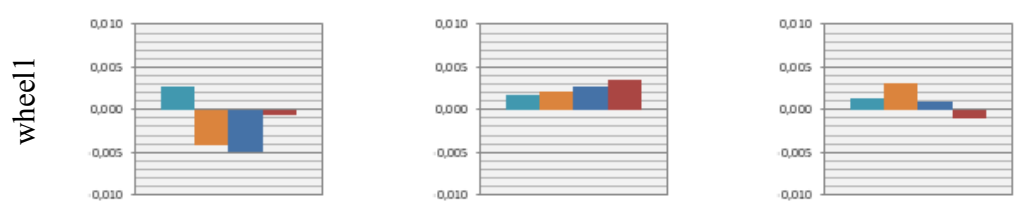

arch $10 \%$

arch $20 \%$

- Arch $30 \%$

arch $40 \%$
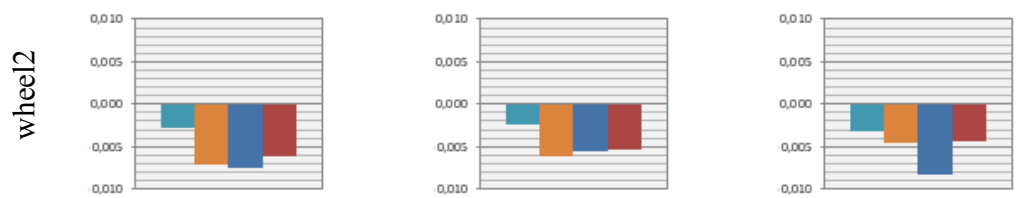

arch $10 \%$

arch $20 \%$

- Arch $30 \%$

arch $40 \%$

Fig. 8. Influence of wheel arch morphing on drag coefficient (compared to $0 \%$ arch height) 


\section{CONCLUSION}

A study of the influence of different parameters defining wheel geometry and rotation has been demonstrated in this work. While many previously done works focus on CFD methodology and local aerodynamic improvements, this investigation provides information of the influence of basic design parameters for electric vehicle.

All the simulations have been performed on full car in aerodynamic virtual wind tunnel. It has been concluded that at early design stage of an electric car various parameters should be taken into account in order to suffice the goal drug coefficient value. The use of scale resolving approaches has been avoided to save the computational costs and time for decision making. The use of RANS simulations on highly detailed mesh allowed to perform extensive parametric investigation within the projects deadlines and providing the drag coefficient with the accuracy of up to 200 drag counts, which is sufficient at early design stages.

The simulation results have demonstrated that consideration of the design of rims is important even in the early phase as it can significantly affect the drag coefficient. Attention should be paid to cases with open rim designs, as these can significantly contribute to aerodynamic resistance because of flow separation. Moreover, simulations should be performed with goal velocity and wheel rotation speed as velocity range in the simulation can also slightly change the drag coefficient. The considered adjustments of ground clearance may help the designer to find balance between comfort of driver and passenger seating position and target drag value. The overall trend of increasing drag with increased ground clearance has been proved.

Modification of wheel arch design can also help in minimizing drag coefficient, but less significantly than other aforementioned design parameters. Moreover, the resulting aerodynamic drag is case-sensitive and depends on combination of wheel arch and rim designs and no clear trends are evident.

Overall, it can be concluded that simulations should be performed with full vehicle setup with car surface, wheel arches and rims in order to produce more reliable results to be used when making design decisions.

\section{References}

[1] T. Hobeika, S. Sebben, "Tyre Pattern Features and Their Effects on Passenger Vehicle Drag," SAE Int. J. Passeng. Cars - Mech. Syst., 11(5), 401-413 (2018)

[2] A. Wäschle. "The Influence of Rotating Wheels on Vehicle Aerodynamics -Numerical and Experimental Investigations", Technical Paper 2007-01-0107, SAE International (2007)

[3] R. Lewis, M. Cross, and D. Ludlow, "The influence of rotating wheels on the external aerodynamic performance of a vehicle", International Vehicle Aerodynamics Conference, 161 - 174 (2014)
[4] J. Wiedemann, "The Influence of Ground Simulation and Wheel Rotation on Aerodynamic Drag Optimization Potential for Reducing Fuel Consumption", Technical Paper 960672, SAE International (1996)

[5] G. Wickern, K. Zwicker , and M. Pfadenhauer, "Rotating Wheels - Their Impact on Wind Tunnel Test Techniques and on Vehicle Drag Results", SAE Technical Paper 970133 (1997)

[6] L. Haag, T. Blacha, T. Indinger, "Experimental Investigation on the Aerodynamics of Isolated Rotating Wheels and Evaluation of Wheel Rotation Models Using Unsteady CFD" International Journal of Automotive Engineering, 8 Issue 1, 7-14 (2017)

[7] E. Rajaratnam, D. Walker, "Investigation of Wheelhouse Flow Interaction and the Influence of Lateral Wheel Displacement", Energies, 12, 3340 (2019)

[8] ANSYS Inc., ANSYS CFX Modeling Guide (2016)

[9] T. Hobeika, S. Sebben, L. Löfdahl, "Study of different tyre simulation methods and effects on passenger car aerodynamics", International Vehicle Aerodynamics Conference, 187-195 (2014)

[10]T. Hobeika, S. Sebben, "CFD investigation on wheel rotation modelling", Journal of Wind Engineering and Industrial Aerodynamics, 174, 241-251 (2018)

[11]T. Hobeika, S. Sebben, and C. Landstrom, "Investigation of the Influence of Tyre Geometry on the Aerodynamics of Passenger Cars," SAE Int. J. Passeng. Cars - Mech. Syst., 6(1), 316-325 (2013)

[12] A.C. Uriegas, "CFD Investigation on Sliding Mesh as a Method to Model Wheel Rotation - Implementation and Analysis on Different Rims" (2018)

[13]T. Wolf, "The aerodynamic development of the new Porsche Cayenne", Journal of Automobile Engineering, 234, 390-408 (2019)

[14] Alvaro Andres Pena, David Fernando Romero, Sergio Raul Rivera Rodriguez,"Generation and Demand Scheduling in a Micro-grid with Battery-based Storage Systems, Hybrid Renewable Systems and Electric Vehicle Aggregators", WSEAS Transactions on Power Systems, pp. 8-23, Volume 14, 2019

[15]Lucjan Setlak, Rafal Kowalik, "The Dynamics of the Movement of Formations of Unmanned Aerial Vehicles", WSEAS Transactions on Applied and Theoretical Mechanics, pp. 192-197, Volume 14, 2019, Art. \#21,

[16] Lucjan Setlak, Rafal Kowalik, "The Dynamics of Group Flights of an Unmanned Aerial Vehicle" WSEAS Transactions on Applied and Theoretical Mechanics, pp. 129-139, Volume 14, 2019,

[17]Lucjan Setlak, Rafal Kowalik, "lgorithm Controlling the Autonomous Flight of an Unmanned Aerial Vehicle based on the Construction of a Glider", WSEAS Transactions on Applied and Theoretical Mechanics, pp. 56-65, Volume 14, 2019,

\section{Creative Commons Attribution License 4.0 (Attribution 4.0 International, CC BY 4.0)}

This article is published under the terms of the Creative Commons Attribution License 4.0

https://creativecommons.org/licenses/by/4.0/deed.en_US 